\title{
Impacto de la Ayuda Financiera en la Persistencia: el Caso de la Universidad de Chile
}

\author{
Impact of Financial Aid on Persistence: the Case of the \\ University of Chile
}

\author{
Macarena Alarcón Valenzuela *1 \\ María Verónica Santelices ${ }^{2}$ \\ Catherine L. Horn ${ }^{3}$ \\ Pablo González Soto ${ }^{4}$ \\ ${ }^{1}$ Universidad de O’Higgins, Chile \\ ${ }^{2}$ Pontificia Universidad Católica de Chile, Chile \\ ${ }^{3}$ Universidad de Houston, Estados Unidos \\ ${ }^{4}$ Universidad de Chile, Chile
}

\begin{abstract}
Introducción: la literatura internacional acerca de la persistencia en la educación superior sugiere que la ayuda financiera entregada a los estudiantes afecta positivamente sus resultados académicos. Sin embargo, en Chile existe escasa investigación respecto a este tema, y en particular no se diferencian los efectos de los distintos tipos de ayuda. De acuerdo a esto, el presente estudio tiene por objetivo explorar en qué medida los diferentes tipos y cantidades de ayuda financiera impactan la probabilidad de persistencia de los estudiantes de la Universidad de Chile. Métodos: se utilizó el modelo cuantitativo de supervivencia para el análisis de eventos históricos, el cual permite, a partir de datos longitudinales, estudiar cómo distintos factores se relacionan con la aparición de un evento en diferentes momentos del tiempo y determinar si estos tienen efectos que cambian en función del tiempo. Las variables utilizadas fueron académicas (carrera, notas, etc.), financieras (tipo de ayuda, montos, etc.) y socio-económicas (región de origen, tipo de establecimiento de educación secundaria, etc.). La información se obtuvo de tres bases de datos de la Universidad de Chile y se analizaron las cohortes de ingreso 2009 y 2010. Resultados y Discusión: los resultados muestran que el otorgamiento de una combinación de becas y préstamos estatales, institucionales $\mathrm{y} / \mathrm{o}$ de origen privado aumenta la probabilidad de permanecer en la Universidad. En particular, se destaca que las becas de mantención tienen un mayor efecto que las ayudas de arancel, y, a su vez, el crédito tiene un mayor impacto que las becas.
\end{abstract}

Palabras clave: Ayuda financiera; Persistencia; Equidad; Políticas públicas; Educación superior; Becas; Matrícula.

Introduction: the international literature on persistence in higher education suggests that students' financial aid affects positively their academic results. However, in Chile and in Latin America in general, there is little research on this issue, and in particular the effects of different types of aid are not differentiated. According to this, the present study has the objective of exploring to what extent the different types and amounts of financial aid impact the probability of persistence of the students of the University of Chile. Methods: the quantitative survival model was used for the analysis of historical events, which allows, from longitudinal data, to study how different factors are related to the appearance of an event at different moments of time and to determine if these have effects that change as a function of time. The variables used were academic (career, grades, etc.), financial (type of aid, amounts, etc.) and socio-economic (region of origin, type of secondary education institution, etc.). The information was obtained from three University of Chile databases; the 2009 and 2010 income cohorts were analyzed. Results and Discussion: the results show that granting a combination of scholarships and state, institutional and / or private loans increases students' probability of persisting in the University. In particular, it is highlighted that grants that cover cost of living expenses have a greater effect than tuition grants, and, in turn, credit has a greater impact than scholarships.

Keywords: Student financial Aid; Academic persistence; Educational equity; Public policy; Higher education; Scholarships; Enrolment.

*Contacto: macarena.alarcon@uoh.cl

issn: 1989-0397

www.rinace.net/riee/

https://revistas.uam.es/riee
Recibido: $\quad 28$ de abril de 2018

$1^{\text {a }}$ Evaluación: 19 de agosto de 2018

$2^{\text {a }}$ Evaluación: 18 de septiembre de 2018

Aceptado: $\quad 21$ de septiembre de 2018 


\section{Introducción}

Chile está viviendo un proceso de reforma educacional histórico impulsado por los movimientos estudiantiles-sociales de los últimos años. A partir de esto, se ha generado un debate en la sociedad chilena acerca de qué modificaciones se deben introducir al sistema educacional y cómo éstas impactarán en el largo plazo.

A nivel de Educación Superior, una de las discusiones contingentes es sobre cuál (es) debe(n) ser el(los) mecanismo(s) de financiamiento para ayudar a los estudiantes a completar sus estudios. Estos cuestionamientos no son nuevos. En los últimos años, tanto el gobierno como las propias universidades han definido e implementado distintas iniciativas para asegurar que los estudiantes puedan acceder y permanecer en el sistema.

Las últimas cifras indican que el gasto público en transferencias por becas y créditos para estudiantes de Educación Superior ha aumentado en un 219,3\% en el período 2009-2015². Esto, junto a otros factores, ha permitido que en los últimos 30 años el sistema de educación superior haya experimentado un crecimiento significativo, pasando de una matrícula de pregrado de 600 mil estudiantes en el año 2005 a una matrícula de más de 1 millón cien mil estudiantes el año $2015^{2}$, y que por consiguiente se haya incrementado el número de estudiantes titulados. De acuerdo a OECD (2009), la educación superior en Chile pasó de ser un sistema de elite a uno masivo, lo cual trajo consigo un cambio en el perfil y en las necesidades de los estudiantes.

En cuanto a graduación, el sistema universitario chileno presenta una tasa de graduación significativamente menor que el promedio de los países de la OCDE $(24 \%$ y $40 \%$, respectivamente). No ocurre lo mismo con el nivel técnico profesional (22\%) que, si bien su tasa de graduación es inferior a la educación universitaria chilena, es ampliamente superior al promedio de la OCDE (11\%) (OCDE, 2009). De acuerdo a esto, SIES (2014c) muestra que más del 50\% de quienes se matriculan en la educación superior no concluyen el programa en el que se matricularon inicialmente. Esta situación genera importantes pérdidas de eficiencia para el Estado y las instituciones, así como una disminución de oportunidades e ingresos para los estudiantes y sus familias.

Lo anterior pone de manifiesto que, si bien el incremento de inversión en ayuda financiera para los estudiantes ha permitido aumentar la cobertura, las tasas de deserción aun podrían disminuir sustancialmente, y las de graduación aumentar. Cabe preguntarse entonces: ¿cuánto contribuyen las becas y créditos a que los estudiantes puedan permanecer en la educación, egresar y titularse?

En este contexto, el presente estudio cuantitativo buscar explorar en qué medida los diferentes tipos y cantidades de ayuda financiera impactan la probabilidad de persistencia de los estudiantes de la Universidad de Chile, y además establecer, a partir de los resultados obtenidos, recomendaciones para la política de financiamiento de educación superior a nivel institucional y nacional. Cabe señalar que la Universidad de Chile fue escogida pues es la Universidad pública más antigua, de mayor tradición y una de las que tiene los más altos estándares de calidad en Chile según la Comisión Nacional de Acreditación, la cual le otorgó la acreditación por el periodo máximo ( 7 años en las 5 áreas

\footnotetext{
${ }^{1}$ Fuente: Portal Beneficios Estudiantiles, Ministerio de Educación.

${ }^{2}$ Fuente: Consejo Nacional de Educación.
} 
evaluadas) y el ranking internacional de Shanghai, el cual la sitúa, en el año 2018, en el primer lugar en 8 áreas a nivel latinoamericano.

$\mathrm{Al}$ respecto, los resultados muestran que el otorgamiento de combinaciones de becas y préstamos estatales, institucionales y/o externas no estatales a los estudiantes aumenta su probabilidad de permanecer en la Universidad. En particular, se destaca que las becas de mantención tienen un mayor efecto que las ayudas de arancel, y, a su vez, el crédito tiene un mayor impacto que las becas.

En las primeras dos secciones de este artículo, y como forma de contextualizar la investigación, se hace una revisión de la literatura existente acerca de este tema y se entregan los principales antecedentes respecto del sistema chileno de educación y de la Universidad de Chile. A continuación, se presenta en detalle la metodología utilizada, y finalmente se dan a conocer los resultados obtenidos y las conclusiones correspondientes.

\section{Revisión de la literatura y fundamentación teórica}

Si bien aún es incipiente, la evidencia empírica internacional y chilena apunta mayoritariamente a un efecto positivo de la ayuda financiera en la permanencia de los estudiantes en la educación superior, y algunos estudios identifican diferencias de impacto entre los distintos instrumentos de apoyo.

En particular, los estudios estadounidenses se enfocan, en su mayoría, en determinar el impacto de la ayuda de acuerdo a si esta fue otorgada por el mérito o la necesidad del estudiante. Otros análisis exploran específicamente como las ayudas en conjunto afectan a los estudiantes según su nivel socio-económico. Respecto de los primeros, Dynarski (2003) mostró que la eliminación del programa histórico de ayuda de Estados Unidos "Social Security Student Benefit Program" en 1982 redujo las probabilidades de asistencia y finalización de los estudiantes en más de un tercio. En esta misma línea, Bettinger (2004) estimó los efectos de las Becas Pell (beneficio otorgado a los estudiantes por su condición socio-económica) sobre la retención estudiantil. Los resultados que obtuvo sugieren que este beneficio reduce las tasas de deserción. Asimismo, Goldrick-Rab, Harris, Kelchen y Benson (2012) examinaron el impacto de un programa privado de ayuda financiera que se otorga según las necesidades de los estudiantes en trece universidades públicas de Wisconsin y en cuatro cohortes de estudiantes. Sus resultados indican que la subvención aumentó la finalización de estudios y las tasas de reinscripción para un segundo año de universidad. En específico, observó que un aumento de 1.00o USD en la ayuda financiera recibida durante el primer año de universidad se asoció con un aumento de 2,8 a 4,1 puntos porcentuales en las tasas de inscripción para el segundo año.

Recientemente Gross, Hossler, Ziskin y Berry (2015) sugieren, a partir de un análisis que compara la probabilidad de desertar de estudiantes que recibieron ayuda institucional por su mérito académico con la de aquellos que la recibieron de acuerdo a su situación socioeconómica, que un aumento en la cantidad de ayuda basada en requisitos socioeconómicos tiene efectos positivos en la retención de los estudiantes, pero que no ocurre lo mismo con la ayuda estudiantil entregada de acuerdo a requisitos académicos (mérito), lo cual plantea la idea de que se podrían estar destinando recursos a estudiantes que de todos modos permanecerían en la Universidad (independientemente de la ayuda que recibieran), y que este tipo de ayuda estaría fomentando la estratificación social y aumentando las desigualdades, pues, en general la ayuda basada en el mérito es entregada 
a los estudiantes que provienen de los estratos sociales más altos. En la misma línea, Bettinger (2015) a través de un experimento natural, estima los efectos de las políticas de ayuda basadas en necesidad en las tasas de persistencia de los primeros años de la universidad en Ohio. Sus resultados indican que las tasas de abandono de estos estudiantes disminuyeron un $2 \%$ como resultado del programa. El programa también aumentó la probabilidad de que los estudiantes asistieran a universidades de 4 años, en vez de instituciones que ofrecen programas más cortos, y de que aumentaran sus calificaciones de primer año. Goldrick-Rab, Kelchen, Harris y Benson (2016), mediante un experimento aleatorio, estimaron el impacto de un programa privado de becas otorgadas a los estudiantes de familias de bajos ingresos en su persistencia y la obtención de un título en 13 universidades públicas de Wisconsin. Los resultados indican que ofrecer a los estudiantes subsidios adicionales incrementa las probabilidades de obtener una licenciatura en cuatro años. Respecto del análisis según las diferencias por nivel socioeconómico, Chen y Desjardins (2008) detectaron que existe una brecha entre las tasas de abandono de los estudiantes de bajos ingresos y las de sus pares de mayores ingresos, y sugieren que algunos tipos de ayuda están asociados con un menor riesgo de deserción.

En contraste a lo señalado anteriormente, en Alemania se introdujeron aranceles en la educación superior. Es decir, pasaron de un sistema de gratuidad a uno que contempla copago por parte de los estudiantes. Bruckmeier y Wigger (2014) determinaron que este cambio no tuvo un efecto negativo en la matrícula agregada, sin embargo, no se ha estudiado si esto afectó la persistencia de los estudiantes en la educación superior. En una línea similar, Rozada y Menendez (2002), a partir de un estudio comparativo entre las universidades públicas y privadas en Argentina, detectaron que al no existir cobros en las instituciones públicas los estudiantes pobres eran excluidos de la educación superior y los estudiantes que asistían a las instituciones privadas eran similares a los que asistían a las instituciones públicas. De acuerdo a esto, sugirieron que la equidad y eficiencia del sistema se podía mejorar mediante el cobro de tasas de matrícula y la introducción de becas selectivas y préstamos estudiantiles a fin de atraer a los estudiantes más talentosos de familias pobres.

Además, y como un tema atingente al otorgamiento de beneficios a los estudiantes, Barr, Chapman, Dearden y Dynarski (2017) analizaron el diseño de los préstamos estudiantiles en Estados Unidos y como se podían mejorar a partir de la experiencia de Australia e Inglaterra, lo cual sugiere que el diseño de los beneficios es relevante para que estos sean sustentables en el tiempo y cumplan el objetivo de ayudar a la persistencia de los estudiantes.

De acuerdo a lo anterior, es posible considerar que las ayudas focalizadas en los estudiantes de menores ingresos impactan en su persistencia debido a que cubren costos que las familias no podrían cubrir de otra forma, sin embargo, esto no es necesariamente generalizable a la realidad chilena.

A nivel nacional, MINEDUC (2012) presenta un análisis transversal del fenómeno de la deserción en educación superior, en el cual destaca que vale la pena investigar en mayor detalle las relaciones y asociaciones entre los distintos instrumentos de beneficios estudiantiles y su impacto en la retención, y concluye que los beneficios estudiantiles están asociados a una menor deserción. En esta línea, Horn, Santelices y Catalán (2014) identificaron que las ayudas estudiantiles institucionales y gubernamentales contribuyen positivamente a la persistencia de los estudiantes de la Pontificia Universidad Católica de 
Chile y Mizala (2011), a partir de un estudio focalizado en los estudiantes chilenos de pedagogía, concluye que alumnos que reciben algún tipo de beneficio estudiantil, ya sea beca o crédito, en particular el CAE, tienen una probabilidad más baja (entre 60\% y 80\%) de desertar con respecto a alumnos sin beneficios.

En cuanto a los tipos de ayuda, Barrios, Paredes y Meneses (2011) concluyen que Crédito con Aval del Estado (CAE) es más eficaz que el Crédito Fondo Solidario (FSCU) y que el Crédito Fondo Solidario (FSCU) es más eficaz que las becas en la reducción de la probabilidad de deserción. Por otro lado, Rau, Rojas y Urzúa (2011) estudiaron el fenómeno poniendo foco en el impacto del Crédito con Aval del Estado (CAE). En su estudio concluyen que el CAE reduce las probabilidades de deserción en primer año y que es más efectivo reduciendo la deserción de los estudiantes provenientes de hogares de menores ingresos. Recientemente, y de forma concordante, Bordón, Canals y Rojas (2015), a través de un estudio para determinar la probabilidad de retención de los estudiantes universitarios en la misma carrera e institución al segundo año, determinaron que contar con Crédito con Aval del Estado aumenta en alrededor del 4\% la probabilidad de retención, mientras que tener Crédito Solidario aumenta en un 1,75\% la probabilidad de retención.

No obstante, la relevancia y aporte de estos hallazgos, la literatura chilena no ha abordado el impacto de las ayudas institucionales y su posible sinergia con el resto de las ayudas financieras estudiantiles. Debido a su importancia y alcance, en este artículo se aborda en profundidad el rol de las ayudas estudiantiles, particularmente las institucionales, en la persistencia de los alumnos que asisten a una universidad selectiva en Chile, la Universidad de Chile.

\section{Antecedentes}

En los párrafos siguientes se muestran los principales antecedentes respecto de la ayuda estudiantil en Chile, cifras de inversión en educación y tasas de retención del sistema universitario chileno y de la Universidad de Chile, en particular.

\subsection{Sistema de ayuda financiera estudiantil en Chile}

Hasta el año 2015, período analizado en este artículo, la matriz de financiamiento a la cual podían acceder los estudiantes del sistema de educación superior chileno estaba conformada principalmente por créditos y becas, los cuales contribuían a cubrir el costo de arancel de las respectivas carreras (pago anual). Las becas correspondían a una transferencia directa y los créditos eran préstamos que los estudiantes debían retribuir, con una tasa de interés regulada, una vez egresados. Además, existían becas para cubrir el costo de vida (mantención) y de alimentación de los estudiantes. Tanto las becas como los créditos eran otorgados por el estado, las propias instituciones y algunas organizaciones externas (fundaciones, municipalidades, etc.), y se entregaban de acuerdo al nivel socioeconómico del estudiante y/o al cumplimiento de algunos requisitos académicos.

El nivel socioeconómico se determinaba partir del quintil de ingreso per cápita familiar registrado en la Universidad por su equipo de asistentes sociales. Los parámetros de los quintiles (valores mínimos y máximos) eran determinados anualmente por el Ministerio de Desarrollo Social el cual, a través de una encuesta nacional, conformaba cinco grupos que diferenciaban a la población chilena por sus niveles de ingresos (total de dinero que aporta el o los sostenedores de un hogar dividido por el número de miembros de éste), siendo el quintil 1 el grupo que recibía menos ingresos y el quintil 5 el que recibía más 
ingresos (quintil: $20 \%$ de la población). En el caso de los requisitos académicos estos principalmente eran el puntaje obtenido en la PSU (prueba de selección universitaria) y/o las notas obtenidas en la educación secundaria.

La renovación de los beneficios, se realizaba anualmente en cada Universidad y en general se le solicitaba al estudiante acreditar el mismo nivel socioeconómico determinado en la asignación inicial y/o cumplir requisitos académicos, como aprobar un cierto porcentaje de asignaturas en su carrera. Si la situación socioeconómica del estudiante cambiaba se le reasignaban los beneficios (por ejemplo, podía pasar de beca a crédito). Las becas, en general, se otorgaban por la duración formal de la carrera y los créditos se entregaban hasta que el estudiante egresara, con un tope máximo de años.

Hoy, si bien siguen existiendo becas y créditos, los alumnos con ingresos familiares dentro de los seis deciles de ingresos más bajos pueden estudiar de manera gratuita en universidades e instituciones técnicas que cumplan con los requisitos de acreditación, de admisión y no lucro establecidos por esta ley.

Hasta hoy en día, el proceso de asignación y renovación de beneficios estudiantiles (becas, créditos y gratuidad) comienza tras la postulación de los alumnos a través del Formulario Único de Acreditación Socioeconómica (FUAS), y culmina cuando MINEDUC, a partir de una revisión exhaustiva de la información de cada estudiante (ingresos económicos de su hogar, resultados académicos, tipo de institución y carrera elegida, obtención de algún otro beneficio de MINEDUC o título profesional, etc.), asigna el mejor paquete de beneficios a cada estudiante.

Estas ayudas son fundamentales para que los estudiantes chilenos que provienen de sectores menos acomodados inicien y desarrollen sus estudios. Sin embargo, factores como la brecha que se ha generado entre los aranceles de referencia ${ }^{3}$ y los aranceles reales para el caso de becas y créditos, y las necesidades de mantención y alimentación no cubiertas por el Estado han hecho que el aporte complementario que realizan las Instituciones de Educación Superior en estas materias se vuelva relevante y crítico, pues en la práctica, los estudiantes financian sus estudios a través de combinaciones de préstamos y becas estatales e institucionales.

\subsection{Sistema de acceso a las universidades chilenas}

Para acceder al sistema universitario chileno, existen dos vías: la prueba de selección universitaria y los sistemas especiales de admisión definidos por cada institución. La prueba de selección universitaria es una batería de pruebas estandarizadas y su puntuación tiene un valor entre 150 a 850 puntos. Todas las universidades adscritas a esta modalidad de selección (universidades públicas y un subconjunto de universidades de carácter público) establecen, para cada programa de pregrado ofrecido, vacantes y ponderaciones para las pruebas y la concentración de notas de enseñanza media. La mayoría de las instituciones requieren un promedio de 450 puntos entre las pruebas de lenguaje y matemática como el puntaje mínimo para postular a una carrera (hay instituciones que definen un puntaje más alto).

\footnotetext{
${ }^{3} \mathrm{El}$ “Arancel de Referencia” es un valor, fijado todos los años el Ministerio de Educación, que indica el monto máximo de financiamiento que otorga el gobierno (crédito y/o beca) para un estudiante para cada carrera e institución de educación superior. La diferencia con el arancel real de la carrera debe ser cubierta a través de otra fuente de financiamiento.
} 
Los requisitos y vacantes de los sistemas especiales de admisión, son definidos por cada institución y buscan valorar aptitudes (por ejemplo, deportistas destacados) o lograr equidad y diversidad en los campus universitarios.

En términos de cifras, en el año 2017 ingresaron a través de estos sistemas 113.206 estudiantes, y los años 2009 y 2010 (cohortes de estudio) ingresaron 109.923 y 118.930 estudiantes respectivamente.

En general, las instituciones fijan un valor anual para el arancel y matrícula de la carrera. El valor de matrícula se paga al inicio del año académico y el arancel se puede pagar en cuotas. En el caso de que al estudiante se le asignen becas y/o crédito, éstos se le descuentan del total de los costos fijados por la Universidad (solo debe pagar la diferencia, si es que la hubiese).

\subsection{Universidad de Chile}

La Universidad de Chile (UCH) es la universidad pública4 de mayor reconocimiento y la más antigua del país (1842). Cuenta con 17 facultades e institutos y una matrícula de pregrado de aproximadamente 30.000 estudiantes, de los cuales el $41 \%$ pertenece a los dos

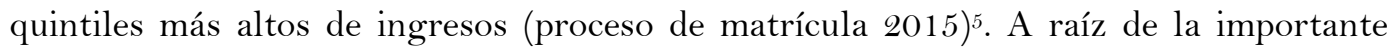
presencia de los quintiles de más altos ingresos, la UCH ha ido consolidado paulatinamente una política universitaria en torno a la equidad, traducida en la implementación de los siguientes elementos: sistema de admisión (SIPEE: Sistema Ingreso Prioritario de Equidad Educativa), que considera variables distintas al puntaje PSU para acceder a sus programas de formación e introduce alternativas de financiamiento y acompañamiento académico para los estudiantes de mayor vulnerabilidad; y la vía de ingreso Escuela de Desarrollo y Talentos. Junto con lo anterior, la $\mathrm{UCH}$ ha desarrollando un sistema complementario de beneficios que ayudan al estudiante a sortear costos de mantención y brecha entre el arancel de referencia y el real.

El año 2013 se adjudicaron 10.587 becas de arancel (internas y externas) y 47.839 becas de mantención. Respecto del año 2010, estas cifras representan un aumento de beneficiados del 53\% (variación monto adjudicado: 113,7\%), y 16,2\% (variación monto adjudicado: 33,3\%) respectivamente (Dirección de Bienestar Estudiantil UCH, 2014).

En cuanto a retención, la tasa promedio de primer año para la cohorte de ingreso 2013 es de $84,3 \%$ (Universidad de Chile, 2013a). Esta se ha mantenido sin variaciones significativas de un año a otro, pero muestra una leve tendencia positiva en el período 2008-2013. La UCH retuvo en promedio al $84,8 \%$ de sus estudiantes de primer año ingresados el 2008 y el $89,9 \%$ de los ingresado el $2013^{6}$.

Estas cifras se han transformado en una preocupación institucional permanente, pues si bien se han incrementado los recursos para becas y créditos, esto no se ha visto reflejado en una mejora de las tasas de retención. Su análisis y respectivo plan de acción se enmarcan dentro del desarrollo del Modelo de Desarrollo Integral del Estudiante (MDIE), el cual busca contribuir a la igualdad de oportunidades de los alumnos a través de tres dimensiones: calidad de vida, aprendizaje y enseñanza (Universidad de Chile, 2013b).

\footnotetext{
${ }^{4}$ Fuente: Ranking de Shangai 2014.

${ }^{5}$ Fuente: Dirección de Bienestar Estudiantil Universidad de Chile.

${ }^{6}$ Fuente: Consejo Nacional de Educación.
} 


\section{Preguntas de investigación}

El estudio buscar responder la siguiente pregunta: ¿En qué medida los diferentes tipos y cantidades de ayuda financiera impactan la probabilidad de persistencia de los estudiantes de la Universidad de Chile?

\section{Metodología}

\subsection{Fuentes de información}

Se utilizaron tres bases de datos de la Universidad de Chile como fuentes de información: registros de admisión y matrícula (carrera y rendimiento académico), registros de ayuda financiera (quintil, montos, tipos y fuente de ayuda) y los registros del proceso de admisión PSU (puntajes PSU, género, tipo de establecimiento educacional secundario, rendimiento académico educación secundaria y región de origen).

\subsection{Muestra}

La muestra utilizada considera los estudiantes que ingresaron a la UCH en las cohortes de ingreso 2009 y 2010, a través de cualquier vía de ingreso: PSU, beca excelencia académica (BEA), personas con estudios medios en el extranjero, ingreso prioritario de equidad educativa (SIPEE), ingreso prioritario de equidad de género (PEG), deportistas destacados, estudiantes no videntes y convenios étnicos.

Cabe señalar que se seleccionaron las cohortes 2009 y 2010 ya que preceden a la reforma educacional promulgada en 2012 y a la introducción de la política universitaria de equidad dentro de la Universidad de Chile, lo cual permitirá realizar estudios comparativos futuros; por la disponibilidad de datos de los primeros años de dos cohortes completas; y por que su elección garantizó que los patrones de comportamiento no respondieran a sucesos particulares de un año en estudio determinado ( 2 cohortes).

\subsection{Variables}

Se utilizaron variables nominales y continuas. En el caso de las primeras, se categorizó la variable sexo, región del estudiante (si es de Santiago o regiones), dependencia educacional (define si el establecimiento secundario es Municipal, Subvencionado Particular o Particular Pagado), quintil de ingreso socioeconómico y tipo de carreras (define si es una carrera del área de Artes Liberales, STEM o otra).

En el caso de las variables continuas, se utilizó el promedio de notas de las asignaturas y la proporción de ayuda financiera (según el modelo) sobre el monto del arancel real de la carrera del estudiante (estandarización de los montos de ayuda), pues los costos de arancel y la ayuda, independiente de su origen y tipo, varían según la carrera y año que cursa el alumno. Por ejemplo: dos estudiantes, uno de pedagogía y otro de medicina, podrían recibir $\$ 500.000$ pesos de ayuda, sin embargo, para el primero esto podría significar un $25 \%$ de su pago anual y para el segundo solo un $15 \%$.

\subsection{Modelo}

Para examinar los patrones de persistencia de los estudiantes a través del tiempo se utilizó el modelo cuantitativo de supervivencia para el análisis de eventos históricos (Singer y Willett, 2003), ya que permite, a partir de datos longitudinales, estudiar cómo distintos 
factores se relacionan con la aparición de un evento en diferentes momentos y determinar si estos tienen efectos que cambian en función del tiempo.

Este modelo considera una variable logit de tipo dicotómica. Según lo señalado por Graham, Willet y Singer (2012), se definieron dos estados para esta variable. Estado 1 es cuando un estudiante permanece estudiando en la Universidad y Estado 2 es cuando un estudiante deserta de sus estudios. Además, se estableció como "momento o", el momento en que todos los miembros de la población ocupan uno, y sólo uno de los estados posibles, es decir, cuando todos los estudiantes están "en riesgo" de desertar por primera vez, lo cuál ocurre justo después que se matriculan como estudiantes nuevos en la UCH (enero de cada año). Se seleccionó como unidad temporal el año, y se estudió la evolución de las cohortes 2009 y 2010 durante seis años, desde el año 2009 al año 2014.

Cabe señalar que, a raíz de la naturaleza de los datos disponibles, en lugar de limitar la definición de persistencia a dos puntos estáticos en el tiempo (por ejemplo, del primer al segundo año o del primero al último año), el análisis considera la distribución de los tiempos de supervivencia en todos los años disponibles a lo largo del tiempo (Fox, 2002).

En cuanto al modelo propiamente tal, éste busca determinar el riesgo de deserción (probabilidad) a través de una función logarítmica de dos conjuntos de predictores. El primer grupo son los $\alpha$ multiplicados por $D_{s}$ (indicadores de tiempo), que representa la función de riesgo Logit base para cada año académico. El segundo grupo son los $\beta$ multiplicados por $X_{S}$ (sus respectivos predictores), lo que representa el cambio en la función de riesgo Logit base debido a la variación de los predictores asociados en una unidad (variables independientes). Los regresores incluidos $\left(X_{S}\right)$ pueden ser las variables invariantes en el tiempo (Ej: sexo del estudiante) o variables en el tiempo, tales como la ayuda estudiantil (Ej: becas). La ecuación (1) describe formalmente el modelo usado en este estudio (Chen y DesJardins 2010).

$$
\text { Logit } h\left(t_{i f}\right)=\left[\alpha_{1} D_{1 i f}+\cdots+\alpha_{J} D_{J i J}\right]+\left[\beta_{1} X_{1 i f}+\cdots+\beta_{J} X_{J i J}\right] \text { (1) }
$$

En términos concretos la función Logit determina la probabilidad condicional de que un estudiante abandone sus estudios en el tiempo j, dado que él o ella no ha experimentado este evento antes del tiempo $\mathrm{j}$.

\subsection{Análisis}

En primer lugar, se analiza, mediante estadísticas descriptivas, la evolución de la proporción de estudiantes con ayuda financiera en la Universidad de Chile y sus puntajes de ingreso. En segundo lugar, se estiman modelos (1, 2, 3 y 4) que analizan la influencia de los distintos tipos de ayuda a través de distintos conjuntos de variables predictoras del abandono. El primer modelo analiza las ayudas de arancel versus las de mantención (independientemente si estas fueron otorgadas a través de beca o crédito), el segundo analiza el efecto de las becas versus el crédito (independientemente si su objetivo fue financiar el arancel y/o la mantención), el tercer modelo realiza un análisis más detallado por fuente de financiamiento (estatal versus institucional) sobre la continuidad de estudios, el cuarto modelo es una desagregación por fuente (estatal o institucional) y tipo (crédito o beca), finalmente el quinto modelo presenta la interacción entre ayuda y quintil de ingreso, y el último modelo revisa la probabilidad de persistencia considerando el área disciplinar. 


\section{Resultados}

\subsection{Análisis descriptivo}

El cuadro 1 representa descriptivamente la evolución de las cohortes 2009 y 2010 desglosadas según si recibieron ayuda financiera o no. En general, se observa que al menos un $61 \%$ de los estudiantes de estas cohortes, que persisten en los siguientes años, reciben algún tipo de ayuda financiera. Solo para la cohorte 2009 en el año 2014 se observa un porcentaje menor.

Por otro lado, la admisión de la Universidad de Chile, requiere, según la carrera a la cual el alumno quiere acceder, distintas pruebas del Sistema Único de Admisión Chileno (PSU). En el cuadro 2 se observa, para ambas cohortes, una diferencia de al menos 10 puntos en la puntuación promedio de cada tipo de prueba entre los estudiantes que recibieron ayuda y aquellos que no. Esto se explica fundamentalmente por la correlación positiva que tienen los puntajes obtenidos por el estudiante con su situación socioeconómica.

Cuadro 1. Evolución de la persistencia de los estudiantes categorizados según si recibieron algún tipo de ayuda en el primer año de estudios

\begin{tabular}{ccccc}
\hline \multirow{2}{*}{ PERIODO } & \multicolumn{2}{c}{$\mathbf{2 0 0 9}$} & \multicolumn{2}{c}{ To10 } \\
\cline { 2 - 5 } & $\begin{array}{c}\text { Tue persisten de } \\
\text { la cohorte }\end{array}$ & $\begin{array}{c}\text { Porcentaje de } \\
\text { estudiantes } \\
\text { persistentes con } \\
\text { ayuda sobre el total }\end{array}$ & $\begin{array}{c}\text { Total estudiantes } \\
\text { que persisten de } \\
\text { la cohorte }\end{array}$ & $\begin{array}{c}\text { Porcentaje de } \\
\text { estudiantes } \\
\text { persistentes con } \\
\text { ayuda sobre el total }\end{array}$ \\
\hline 2009 & 4.800 & $61,20 \%$ & 5.109 & $63,50 \%$ \\
2010 & 3.984 & $66,20 \%$ & 4.084 & $67,20 \%$ \\
2011 & 3.560 & $67,50 \%$ & 3.785 & $61,60 \%$ \\
2012 & 3.354 & $62,60 \%$ & 3.489 & $64,80 \%$ \\
2013 & 3.056 & $64,70 \%$ & 2.994 & $65,20 \%$ \\
2014 & 2.167 & $52,20 \%$ & & \\
\hline
\end{tabular}

Fuente: Elaboración propia.

Cuadro 2. Puntajes promedio de los estudiantes según tipo de prueba PSU y si tuvo algún tipo de ayuda en el primer año de estudios

\begin{tabular}{lcccc}
\hline & \multicolumn{2}{c}{$\mathbf{2 0 0 9}$} & & $\mathbf{2 0 1 0}$ \\
\hline & Con Ayuda & Sin Ayuda & Con Ayuda & Sin Ayuda \\
PSU Ciencias & 677,6 & 687,6 & 678,4 & 694,9 \\
PSU Lenguaje y Comunicaciones & 684,4 & 698,1 & 688,1 & 695 \\
PSU Matemática & 681 & 693 & 685,3 & 708,8 \\
PSU Historia y Ciencias Sociales & 688,3 & 698,2 & 692,3 & 698,4 \\
Puntaje notas de enseñanza media & 675,7 & 684,1 & 667,6 & 679,4 \\
\hline
\end{tabular}

Fuente: Elaboración propia.

\subsection{Resultados del Modelo de Supervivencia}

La Tabla 3 muestra los resultados de los modelos analizados. Es importante señalar que cada uno de los modelos considera un tipo particular de categorización de la ayuda financiera otorgada a los estudiantes. Al respecto, se debe considerar que si el valor de Exp (B) es mayor a 1 la asociación es positiva, es decir, la presencia del factor (tipos de ayuda financiera) se asocia a una mayor ocurrencia del evento (persistencia). Por ejemplo, para el presente análisis, el valor del $\operatorname{Exp}(\mathrm{B})$ obtenido en el Modelo 2 para la variable Proporción Ayuda Crédito es de 1.285, lo cual significa que es 1.285 veces más probable que un estudiante que recibe crédito persista por sobre otro que no recibe este tipo de ayuda. 
En términos generales e independiente del tipo y fuente de financiamiento de la ayuda, se observa que un estudiante que recibe ayuda tiene más probabilidades de persistir que uno que no. En particular, el Modelo 1 muestra que la probabilidad de persistir de un estudiante que recibe ayuda para cubrir su arancel y mantención, es mayor que uno que no recibe este tipo de ayuda. Se observa, sin embargo, una mayor relación entre persistencia y la ayuda de mantención $(\operatorname{Exp}(B)=1,968)$ que entre persistencia y la ayuda de arancel.

A partir del Modelo 2, se desprende que la probabilidad de persistir de un estudiante que recibe ayuda como beca o crédito, es mayor que aquella de un estudiante que no recibe ayuda. Al considerar ambos tipos de ayuda y comparar su importancia, se observa una mayor relación entre persistencia y la ayuda recibida en forma de crédito $(\operatorname{Exp}(B)=1,285)$ que entre persistencia y la ayuda recibida en forma de beca.

Cuando se analiza la fuente de ayuda (Cuadro 3: Modelo 3 y 4), se observa que la oportunidad de persistir de un estudiante que recibe ayuda es mayor que uno que no recibe ayuda, independientemente si esta proviene de la propia universidad, del estado o de alguna entidad privada. Al comparar estos tres tipos de financiamiento se observa un mayor coeficiente de la ayuda denominada "otras", la cual agrupa el financiamiento estudiantil otorgado por entidades externas no estatales $(\operatorname{Exp}(B)=1,485)$, como, por ejemplo, la beca que entrega la Municipalidad de las Condes para el arancel y matrícula de un grupo pequeño de estudiantes. En esta línea, el Modelo 4 evidencia una mayor asociación entre persistencia y las becas externas no estatales y las becas entregadas por la Universidad. La menor relación se observa entre persistencia y las becas estatales.

Los resultados del análisis de interacción (Modelo 5) muestran que la probabilidad de persistir de un estudiante que recibe ayuda y pertenece a alguno de los cuatro primeros quintiles es levemente mayor respecto a un estudiante del quintil 5 (grupo de control). Además, se diferencian dos tramos con valores de coeficientes similares: quintil 1-2 y quintil 3-4. 
Cuadro 3. Resultados por modelo

\begin{tabular}{|c|c|c|c|c|c|}
\hline MODELO & $\begin{array}{l}\text { 1. AYUDA ARANCEL - } \\
\text { MANTENCIÓN }\end{array}$ & 2. AYUdA BECA - CRÉDITO & $\begin{array}{c}\text { 3. AYUDA } \\
\text { INSTITUCIONAL - } \\
\text { ESTATAL - OTRAS }\end{array}$ & $\begin{array}{l}\text { 4. AYUDAS DESAGREGADAS } \\
\text { POR TIPO Y FUENTE DE } \\
\text { FINANCIAMIENTO }\end{array}$ & $\begin{array}{c}\text { 5. INTERACCIÓN ENTRE } \\
\text { QUINTIL Y PROPORCIÓN DE } \\
\text { AYUDA TOTAL }\end{array}$ \\
\hline & -2LL: 765188,976 & -2LL: 764553,666 & -2LL: 764984,318 & -2LL: 763814,229 & -2LL: 765090,44 \\
\hline Variables en la ecuación & $\operatorname{Exp}(B)$ & $\operatorname{Exp}(B)$ & $\operatorname{Exp}(B)$ & $\operatorname{Exp}(B)$ & $\operatorname{Exp}(B)$ \\
\hline Sexo & $0,934(*)$ & $0,940(*)$ & $0,938(*)$ & $0,943(*)$ & $0,936(*)$ \\
\hline Región & 1,009 & 1,009 & 1,004 & 1,010 & 1,005 \\
\hline Quintil (1) & $0,747(*)$ & $0,891(*)$ & $0,792(*)$ & $0,900(*)$ & $0,794(*)$ \\
\hline Quintil (2) & $0,763\left(^{*}\right)$ & $0,911(*)$ & $0,806(*)$ & $0,921(*)$ & $0,833(*)$ \\
\hline Quintil (3) & $0,814 * *)$ & $0,786(*)$ & $0,833(*)$ & $0,779(*)$ & $0,707(*)$ \\
\hline Quintil (4) & $0,848(*)$ & $0,790(*)$ & $0,861(*)$ & $0,785(*)$ & $0,738(*)$ \\
\hline Dependencia Educacional (1) & 0,953 & 0,964 & 0,955 & 0,956 & 0,976 \\
\hline Dependencia Educacional (2) & 0,958 & 0,961 & 0,962 & 0,958 & 0,980 \\
\hline Promedio PSU & 1,000 & 1,000 & $0,999(*)$ & $0,999(*)$ & $1,000(*)$ \\
\hline Puntaje NEM & 1,000 & 1,000 & $1,000(*)$ & 1,000 & 1,000 \\
\hline Promedio Universidad (**) & $1,094(*)$ & $1,097(*)$ & $1,095(*)$ & $1,096(*)$ & $1,095(*)$ \\
\hline Proporción Ayuda Arancel (**) & $1,156(*)$ & & & & \\
\hline \multirow[t]{2}{*}{ Proporción Ayuda Mantención (**) } & 1,968 (*) $^{\prime}$ & & & & \\
\hline & -2LL: 765188,976 & -2LL: 764553,666 & -2LL: 764984,318 & -2LL: 763814,229 & -2LL: 765090,44 \\
\hline Proporción Ayuda Crédito (**) & & $1,285(*)$ & & & \\
\hline Proporción Ayuda Beca $(* *)$ & & $1,090(*)$ & & & \\
\hline Proporción Ayuda Institucional (**) & & & $1,397(*)$ & & \\
\hline Proporción Ayuda Estatal (**) & & & $1,160(*)$ & & \\
\hline Proporción Ayuda Otras (***) & & & $1,485(*)$ & & \\
\hline Proporción Ayuda Beca Institucional (**) & & & & $1,418(*)$ & \\
\hline Proporción Ayuda Beca Estatal (***) & & & & $1,050(*)$ & \\
\hline Proporción Ayuda Crédito Estatal (**) & & & & $1,302(*)$ & \\
\hline Proporción Ayuda Beca Otras (**) & & & & $1,528(*)$ & \\
\hline Proporción Ayuda Total (**) & & & & & $1,093(*)$ \\
\hline \multicolumn{6}{|l|}{ Proporción Ayuda Total *Quintil (**) } \\
\hline Proporción Ayuda Total *Q Quintil (1) (**) & & & & & $1,056(*)$ \\
\hline Proporción Ayuda Total * Q ${ }^{2}$ uintil (2) (**) & & & & & $1,040\left(^{*}\right)$ \\
\hline Proporción Ayuda Total * Quintil (3) (**) & & & & & $\left.1,139{ }^{*}\right)$ \\
\hline Proporción Ayuda Total *Q Quintil (4) (**) & & & & & $1,140(*)$ \\
\hline \multicolumn{6}{|l|}{ Tipos Carreras Artes Liberales (1) } \\
\hline Tipos Carreras STEM (2) & & & & & \\
\hline Proporción Ayuda Total (**) & & & & & \\
\hline
\end{tabular}

$(*) p<.01 ;(* *)$ Variables que varían en el tiempo.

Fuente: Elaboración propia. 


\section{Discusión de los resultados}

Los resultados apoyan, en muchos aspectos, la literatura disponible acerca de la contribución de la ayuda financiera a la persistencia de los estudiantes. Para ambas cohortes analizadas el otorgamiento a los estudiantes de combinaciones de becas y préstamos estatales, institucionales y/o externas no estatales, se relacionan con mayores probabilidades de persistencia en la Universidad.

Al realizar un desglose más fino, destaca que las ayudas de mantención tienen una relación más fuerte con la persistencia que las ayudas de arancel. Esto probablemente se deba al costo de oportunidad que tienen los estudiantes de menores recursos, pues cuando un estudiante decide continuar sus estudios deja de aportar ingresos a su familia (no trabaja remuneradamente). Además, el estudiante busca no constituir un gasto adicional, de ahí que la ayuda adicional en cuanto a vivienda, alimentación, materiales, etc. sea determinante. Esto es concordante con los resultados de Paredes y Hernández (2007), quienes señalan que las ayudas estudiantiles (becas y créditos) no son suficientes para resolver las restricciones de liquidez de los estudiantes.

Desde otro punto de vista, resalta el tamaño del coeficiente del crédito por sobre las becas, lo cual concuerda con lo señalado por Rolando, Salamanca y Lara (2012) y Santelices, Catalán, Horn y Kruger (2013) acerca de la correlación positiva entre la asignación del CAE y la persistencia de los estudiantes. El primero de estos estudios, establece que los estudiantes con ayuda financiera tienen una mayor persistencia, y aun más, estimaron un mayor porcentaje de persistencia de los estudiantes beneficiarios del CAE en relación a los beneficiarios de becas. En la misma línea, el segundo estudio determinó diferencias por tipo de crédito: alumnos que reciben el crédito Fondo Solidario son 33\% más proclives a persistir hasta el segundo año que alumnos que no lo reciben, mientras que alumnos que reciben el crédito con aval del estado (CAE) son dos veces más propensos a persistir que alumnos que no lo reciben.

Adicionalmente, los hallazgos son coherentes con el estudio evaluativo y descriptivo realizado por World Bank (2011), el cual muestra que, debido a la implementación del CAE, las tasas de deserción entre beneficiados del CAE parecen ser de 3 a 4 veces menores que las tasas generales de deserción. En esta misma línea, Horn, Santelices y Catalán (2014) detectaron, para el caso de la Pontificia Universidad Católica de Chile, que los préstamos otorgados por el Ministerio de Educación contribuyen a la persistencia de los estudiantes del quintil 1 y 2 .

Cuando los datos se desagregan por fuente de financiamiento, la ayuda otorgada por entidades no estatales externas a la universidad es la que está asociada a un mayor diferencial en la probabilidad de permanencia. En general este tipo de ayuda es dirigida a estudiantes con un alto mérito académico y es otorgada por Fundaciones, Municipios y entidades bancarias, entre otras, por montos que varían entre $\$ 245.000$ y $\$ 4.750 .000$, cubriendo de manera total o parcial el arancel del alumno, gastos de mantención o ambos. En segundo lugar, aparece la ayuda institucional, principalmente becas que cubren el saldo que no es cubierto por el Estado. Esto se alinea con el trabajo de Perna, Lundy-Wagner, Yee, Brill y Tadal (2010) que concluyen que la disponibilidad de ayuda institucional junto a la ayuda estatal, contribuyen en forma conjunta a la probabilidad de que los estudiantes de bajos ingresos matriculados en la universidad continúen sus estudios. 
Los resultados obtenidos en el Modelo 5 (modelo de interacción), sugieren la necesidad de profundizar, mediante nuevos estudios, el análisis de la interacción entre ayuda recibida y quintil socioeconómico del estudiante. En especial, es importante considerar que los resultados observados en la interacción del quintil y ayuda financiera en este estudio pueden estar influenciados por los antecedentes académicos de los alumnos de la Universidad de Chile, ya que estos ingresan con un puntaje mínimo ponderado de 600 puntos (UCH es altamente selectiva ${ }^{7}$ ), lo cual condiciona el ingreso de estudiantes de menores recursos a la institución. De confirmarse los resultados de este estudio, sería recomendable revisar la actual focalización de la ayuda financiera en los quintiles de ingreso más bajo para, eventualmente, expandir el apoyo económico a alumnos de quintiles de ingreso más alto.

Por otro lado, los resultados de todas las regresiones realizadas muestran que los estudiantes de sexo masculino, respecto de las estudiantes de sexo femenino, tienen un mayor riesgo de no permanecer en la Universidad (menores oportunidades), lo cual es concordante con lo señalado en dos estudios realizados por SIES (2014a, b). En el primero de éstos se observa que las mujeres tienen mejores tasas de retención de primer año que los hombres a nivel de pregrado, aunque se segmente por tipo de institución, carrera, jornada y/o área del conocimiento. Esto se podría relacionar con la tendencia al empoderamiento femenino en el mundo público, es decir, aumento de su participación en educación tanto secundaria como terciaria, en el ámbito laboral y mundo político (Comunidad Mujer, 2014). Los análisis de SIES (2014b) muestran además una predominancia de la participación femenina en la matrícula y tasas de retención en educación superior.

Igualmente es interesante destacar que algunos de los modelos sugieren que los resultados obtenidos por los estudiantes en la educación media no contribuyen a su persistencia en la Universidad (Puntaje Promedio PSU y Puntaje NEM), lo cuál se asemeja a lo observado por Horn, Santelices y Catalán (2014). Es posible que estos resultados se deban, en parte, al hecho que los estudiantes de la Universidad de Chile se encuentran entre aquellos de mejor desempeño de sus respectivos establecimientos educacionales, lo cuál técnicamente podría estar evidenciando una restricción de rango en algunas de las variables explicativas analizadas.

\subsection{Limitaciones del estudio}

El presente estudio está limitado en varios aspectos. En primer lugar, la composición socioeconómica de la $\mathrm{UCH}$ es distinta a la del resto de las instituciones de educación superior del país (aproximadamente $26 \%^{8}$ pertenece al quintil de ingresos 1 y 2 , y el puntaje promedio PSU de ingreso es $612^{9}$ puntos) por lo que los resultados de este análisis no son generalizables a otras casas de estudios. (Gil, Paredes y Sánchez, 2013).

En segundo lugar, los datos socioeconómicos disponibles para los años 2009 y 2010 son limitados en algunos aspectos. Esto porque, en aquellos años, las Universidades, en conjunto con el MINEDUC, solo evaluaban socioeconómicamente a los estudiantes que requerían algún tipo de ayuda y, además, algunas ayudas pequeñas eran gestionadas

${ }^{7}$ La Universidad de Chile es una de las dos universidades chilenas que exige un puntaje mínimo ponderado de 600 puntos. El resto de las instituciones exige puntajes mínimos más bajos.

${ }^{8}$ Procesos de admisión 2010, 2011 y 2012.

${ }^{9}$ Proceso de admisión 2013. 
directamente por las facultades e institutos, y su información no llegaba a la administración central de la Universidad. A raíz de esto y para efectos del estudio, se consideraron a los estudiantes sin evaluación socioeconómica como estudiantes pertenecientes al quinto quintil.

Un tercer aspecto es que la metodología utilizada no permite establecer relaciones de causalidad debido a la relación entre las características académicas y sociodemográficas con la variable dependiente de interés (persistencia), lo que se conoce como fenómeno de "endogeneidad" en econometría.

Además, existe un cuarto aspecto relacionado con la calidad de los datos disponibles. Las distintas facultades e institutos de la Universidad utilizaban distintas escalas para evaluar a sus estudiantes: escalas alfanuméricas, escalas numéricas de 1 a 7 con distintas formas de aproximación, etc. Este aspecto fue soslayado a través de aproximaciones y transformaciones lineales a fin de elaborar una escala única.

Por último, la diversidad de modalidades en que se imparten las asignaturas (anuales, semestrales, de verano, etc.) no permitió considerar el semestre como unidad de tiempo, pues un porcentaje importante de los estudiantes cursan asignaturas anuales y por tanto su permanencia solo se puede medir al inicio y final del año.

\subsection{Implicancias de política, futuras investigaciones y conclusiones}

El presente estudio analizó en qué medida los diferentes tipos y cantidades de ayuda financiera impactan la probabilidad de persistencia de los estudiantes de la Universidad de Chile. Una mayor comprensión de las variables que aportan al éxito de los estudiantes, en particular de los más vulnerables, puede proporcionar información útil tanto para la toma de decisiones a nivel nacional como institucional.

En términos generales los resultados del estudio señalan que la ayuda financiera se relaciona con una mayor permanencia de los estudiantes, y, por tanto, es conveniente que las universidades analicen cómo podrían aprovechar de mejor manera los recursos que tienen disponibles para disminuir el riesgo de deserción.

Específicamente, los resultados sugieren que las universidades pueden re-organizar las formas y criterios de asignación de las distintas ayudas institucionales con que cuentan, y producir así un aumento de la persistencia de los estudiantes. En este sentido, analizar y rediseñar la sinergia entre la ayuda estatal y la ayuda institucional puede colaborar a aumentar el impacto y alcance de los recursos.

Además, los distintos análisis muestran que la ayuda de mantención tiene un impacto importante, en consecuencia, las políticas universitarias tanto locales como a nivel nacional deberían evaluar la posibilidad de destinar más recursos para alimentación, vivienda y transporte, cubriendo así de forma integral las necesidades del estudiante.

Finalmente, en el futuro, sería interesante profundizar en diversos aspectos. El caso de la Universidad de Chile ofrece oportunidades para analizar si la ayuda financiera puede contribuir a mejorar el rendimiento académico, la graduación, los tiempos de titulación y/o los tiempos de inserción en el mundo laboral. Por otro lado, sería interesante realizar estudios similares, pero considerando un conjunto más amplio de instituciones. 


\section{Agradecimientos}

Pablo González agradece el fianciamiento provisto por el proyecto CIE160009 y María Verónica Santelices agradece al proyecto Fondecyt 1160871 y al financiamiento de la Iniciativa Científica Milenio del Ministerio de Economía, Desarrollo y Turismo a través del proyecto Núcleo Milenio Experiencias de los Estudiantes de la Educación Superior en Chile: Por qué y Para qué.

\section{Referencias}

Allison, P. D. (1982). Discrete-time methods for the analysis of event histories. En S. Leinhardt (Ed.), Sociological methodology (pp. 61-98). San Francisco, CA: Jossey-Bass.

Arnau, J. y Arce, C. (1996). Métodos y técnicas avanzadas de análisis de datos en ciencias del comportamiento. Barcelona: Edicions Universitat de Barcelona

Attewell, P., Heil, S. y Reisel, L. (2011). Competing Explanations of Undergraduate Noncompletion. American Educational Research Journal, 48(3), 536-559. https://doi.org/10.3102/0002831210392018

Barr, N., Chapman, B., Dearden, L. y Dynarski, S. (2017). Getting StudentFinancing Right in the US: Lessons from Australia and England. Londres: Centre for Global Higher Education.

Barrios, A., Meneses, F. y Paredes, R. (2011). Financial Aid and University Attrition in Chile. Santiago de Chile: Documento de Trabajo, Pontificia Universidad Católica de Chile.

Bettinger, E. (2004). How financial aid affects persistence. En C. M. Hoxby (Ed.), College choices: The economics of where to go, when to go, and how to pay for it (pp. 207-233). Chicago, IL: University of Chicago Press.

Bettinger, E. (2015). Need-based aid and college persistence the effects of the Ohio College Opportunity Grant. Educational Evaluation and Policy Analysis, 37(1 suppl.), 102S-119S. https://doi.org/10.3102/0162373715576072

Bordón, P., Canals, C. y Rojas S. (2015). Retención en los programas e instituciones de educación superior Nueva evidencia para Chile. Revista Estudios de Política Educativa, 2, 174-2 14.

Bruckmeier, K. y Wigger, B. (2014). The effects of tuition fees on transition from high school to university in Germany. Economics of Education Review, 41, 14-23. https://doi.org/10.1016/j.econedurev.2014.03.009

Canales, A. y de los Ríos, D. (2007). Factores explicativos de la deserción universitaria. Revista Calidad en la Educación, 26, 173-201.

Chen, R. y DesJardins, S. L. (2008). Exploring the effects of financial aid on the gap in student dropout risks by income level. Research in Higher Education, 49(1), 1-18.

Chen, R. y DesJardins, S. L. (2010). Investigating the impact of financial aid on student dropout risks: Racial and ethnic differences. Journal of Higher Education, 81(2), 179-208. https://doi.org/10.1353/jhe.0.0085

Comisión de Financiamiento Estudiantil para la Educación Superior (2012). Análisis y Recomendaciones para el Sistema de Financiamiento Estudiantil. Chile: Comisión de Financiamiento Estudiantil.

Comunidad Mujer. (2014). Boletín Mujer y Trabajo: Género y trayectorias profesionales. Serie Comunidad Mujer, 27. Recuperado de https://goo.gl/raH29z 
Cox, D. R., (1972). Regression models and life-tables (with discussion). Journal of the Royal Statistical Society, 34, 187-220.

Deming, D. y Dynarski, S. (2008). The Lengthening of Childhood. Journal of Economic Perspectives, 22(3), 71-92.

División de Educación Superior (2014). Memoria Financiamiento Estudiantil 2014. Santiago de Chile: Ministerio de Educación.

Dynarski, S. (2003). "Does Aid Matter? Measuring the Effects of Student Aid on College Attendance and Completion”. American Economic Review, 93(1), 279-288.

Fox, J. (2002). Cox proportional-hazards regression for survival data. An $r$ and s-plus companion to applied regression. Thousand Oaks, CA: Sage Publications.

Gil. J., Paredes. R. y Sánchez. I. (2013). El Ranking de Notas. Inclusión con Excelencia. Santiago de Chile: Centro de Políticas Públicas.

Graham, S., Willet, J. y Singer, J. (2012). Using Discrete-Time Survival Analysis to Study Event Occurrence. En J. Newsom, R. Jones y S. Hofer (Eds.), Longitudinal Data Analysis: A Practical Guide for Researchers in Aging, Health, and Social Sciences (pp. 329-371). Nueva York, NY: Taylor y Francis Group.

Goldrick-Rab, S., Harris, D. N., Kelchen, R. y Benson, J. (2012). Need-Based Financial Aid and College Persistence: Experimental Evidence from Wisconsin. Madison, WI: Institute for Research on Poverty.

Goldrick-Rab, S., Kelchen, R., Harris, D. N. y Benson, J. (2016). Reducing income inequality in higher education: Experimental evidence on the impact of financial aid on college completion. American Journal of Sociology, 121(6), 1762-1817.

Gross, J., Hossler, D., Ziskin, M. y Berry, M. (2015). Institutional Merit-Based Aid and Student Departure: A Longitudinal Analysis. The Review of Higher Education, 38, 221-250. https://doi.org/10.1353/rhe.2015.0002

Horn, C., Santelices, V. y Catalán, X., (2014). Modeling the Impacts of National and Institutional Financial Aid Opportunities on Persistence at an Elite Chilean University. Higher Education, 68(3), 471-488. https://doi.org/10.1007/s10734-014-9723-3

Intelis y Verde. (2012). Evaluación Final y Propuestas. Evaluación de Impacto de los Programas de Becas de Educación Superior del Ministerio de Educación. Santiago de Chile: Intelis y Verde.

Long, B. T. (2010). Making college affordable by improving aid policy. Issues in Science and Technology, 26(4), 27-38.

Menéndez, J. (2014). Educación Superior en Chile: ¿fin de un ciclo de expansión para las universidades?. Revista Latinoamericana de Educación Comparada, 5(5), 134-148.

MINEDUC. (2012). Serie de Evidencias: Deserción en la educación superior en Chile. Santiago de Chile: MINEDUC.

Mizala, A., Hernández, T. y Makovec, M. (2011). Determinantes de la Elección y Deserción en la Carrera de Pedagogía. En Evidencias para Políticas Públicas en Educación (Ed.), Selección de investigaciones $5^{\circ}$ concurso FONIDE (pp. 165-203). Santiago de Chile: Ministerio de Educación.

Museus, S. D. y Quaye, S. J. (2009). Toward an intercultural perspective of racial and ethnic minority college student persistence. The Review of Higher Education, 33(1), 67-94. 
Organización para la Cooperación y el Desarrollo Económicos (OCDE). (2009). Reviews of national policies for education reviews of national policies for education: Tertiary education in Chile. París: OCDE Ediciones.

Paredes, R. (2013, octubre). ¿Quién debe pagar?. Presentación realizada en el Seminario Financiamiento de la Educación en Chile, Universidad de Chile (Santiago de Chile).

Paredes, R. y Hernández, L. (2007). Restricciones económicas en la decisión de continuar estudios superiores técnicos o profesionales. Calidad en la Educación, 27, 238-261.

Park, J., Denson, N. y Johnson, M. (2014). Examining a financial climate of support: How institutional-level financial aid relates to teamwork, leadership, and critical thinking. Journal of College Student Development, 55(8), 779-794. https://doi.org/10.1353/csd.2014.0086

Perna, L., Lundy-Wagner, V., Yee, A., Brill, L. y Tadal, T. (2010). Showing them the money: The role of institutional financial aid policies and communication strategies in attracting lowincome students. En A. Kezar (Ed.), Recognizing and serving low-income students in higher education (pp. 72-96). Nueva York, NY: Routledge.

Rau, T., Rojas, E. y Urzúa, S. (2011). Restricciones de Crédito y Deserción de la Educación Superior: Evidencia del Caso Chileno. Santiago de Chile: Ediciones de la Universidad de Chile.

Rolando, R., Salamanca, J. y Lara, A. (2012). Retención de 1er año en Educación Superior: Carreras de pregrado. Santiago de Chile: MINEDUC.

Rozada, M. y Menendez, A. (2002). Public university in Argentina: subsidizing the rich? Economics of Education Review, 21(4), 341-351.

San Segundo, M. (2001). Economía de la Educación. Madrid: Editorial Síntesis.

Santelices, V., Catalán, X., Horn, C. y Kruger, D. (2013). Determinantes de Deserción en la Educación Superior Chilena, con Énfasis en Efecto de Becas y Créditos. Informe final: FONIDE F611103.

SIES. (2012). Informe de Titulación. Recuperado de http://www.mifuturo.cl/images/Informes_sies/titulados/titulacion_2012_sies.pdf

SIES. (2014a). Retención en Educación Superior con perspectiva de género. Recuperado de http://www.mifuturo.cl/images/Estudios/Estudios_SIES_DIVESUP/retencion\%20en\% 20educacion\%20superior\%20en\%20perspectiva\%20de\%2ogenero_2014.pdf

SIES. (2014b). Principales indicadores 2014 de Educación Superior en perspectiva de género Matrícula, Titulación y Retención. Recuperado de http://www.mifuturo.cl/images/Estudios/Estudios_SIES_DIVESUP/principales\%20ind icadores\%20de\%20educacion\%20superior\%20en\%20perspectiva\%20de\%20genero\%20matr icula\%20titulacion\%20y\%20retencion_2014.pdf

SIES. (2014c). Retención de Primer Año en Educación Superior - Programas de Pregrado. Recuperado de http://www.mifuturo.cl/images/Estudios/Estudios_SIES_DIVESUP/retencion_primer_ ao_carreras_de_pregrado_2014.pdf

Singer, J. D. y Willett, J. B. (2003). Applied Longitudinal Data Analysis: Modeling Change and Event Occurrence. Nueva York, NY: Oxford University Press.

St. John, E. y Starkey, J. B. (1995). An alternative to net price: Assessing the influence of prices and subsidies on within-year persistence. Journal of Higher Education, 66(2), 156-186.

St. John, E., Cabrera, A., Nora, A. y Asker, E. (2000). Economic influences on persistence reconsidered: How can finance research inform the reconceptualization of persistence 
models? En J. Braxton (Ed.), Reworking the student departure puzzle (pp. 29-47). Nashville, TE: Vanderbilt University Press.

Centro de Microdatos. (2008). Estudio sobre causas de la deserción universitaria. Santiago de Chile: Universidad de Chile.

Unidad de Análisis Institucional. (2013). Anuario 2013. Santiago de Chile: Universidad de Chile.

Prorrectoría. (2013). Memoria 2010-2013 Equidad y Calidad: El compromiso de la Universidad de Chile con el país. Santiago de Chile: Universidad de Chile.

Dirección de Bienestar Estudiantil. (2014). Memoria 2010-2014. Santiago de Chile: Universidad de Chile.

World Bank. (2011). Programa de Crédito con Aval del Estado: Análisis y Evaluación. Santiago de Chile: Sector de Educación, América Latina y el Caribe del Banco Mundial.

\section{Breve CV de los autores}

\section{Macarena Alarcón Valenzuela}

Macarena Alarcón es Directora de Asuntos Estudiantiles en la nueva Universidad pública de O'Higgins desde 2017. Trabajó en la Dirección General de Planificación y Desarrollo de la Universidad Técnica Federico Santa María, y en la Vicerrectoría de Asuntos Académicos y la Vicerrectoría de Asuntos Económicos y Gestión Institucional de la Universidad de Chile. Ella es Ingeniero Civil Industrial de la Universidad Técnica Federico Santa María, y recibió su Maestría en Gestión y Políticas Públicas de la Universidad de Chile. Email: macarena.alarcon@uoh.cl

\section{María Verónica Santelices Etchegaray}

Profesora Asociada de la Facultad de Educación de la Universidad Católica de Chile y afiliada al Núcleo Milenio Experiencias de los Estudiantes de la Educación Superior en Chile. Sus trabajos han sido publicados en revistas especializadas tales como Harvard Educational Review, Educational Psychological Measurement y Higher Education. Sus intereses combinan la medición educacional y las políticas educacionales focalizándose en temas tales como el acceso a la educación superior y la calidad docente. Recibió su Doctorado en Educación de la Universidad de California Berkeley y un Master en Políticas Públicas de la misma universidad. Email:vsanteli@uc.cl

\section{Catherine Horn}

Profesora de Liderazgo Educativo y Estudios de Políticas y Directora Ejecutiva del Instituto de Investigación y Evaluación de Políticas Educativas de la Universidad de Houston. Sus intereses de investigación incluyen las barreras sistémicas al acceso a la universidad para estudiantes tradicionalmente poco representados, las pruebas de altas consecuencias en educacion primaria y secundaria, las política de admisión a la universidad, el uso de los resultados de investigación y los métodos cuantitativos. Email: clhorn2@uh.edu 


\section{Pablo González Soto}

Ph.D. en Economía (1996), University of Cambridge. Director académico del Centro de sistemas públicos del Departamento de Ingeniería Industrial, de la Facultad de Ciencias Físicas y Matemáticas, de la Universidad de Chile. Investigador principal del Centro de investigación para la educación inclusiva, proyecto CIE160009 de la Comisión Nacional de Investigación Científica y Tecnológica. Fue jefe de la división de planificación y presupuesto del ministerio de educación de Chile a mediados de los noventa, y es consultor externo en temas de educación y políticas de infancia de diversos organismos internacionales. Coautor de libros editados por el Banco Mundial, BID, PNUD, UNICEF y OECD, entre otros, así como artículos en revistas en temas de desarrollo y educación que incluyen Habitat International, World Development, e Information Society. Email: pgonzale@dii.uchile.cl 